\title{
SOME FIXED POINT RESULTS FOR $A$-CONTRACTIONS IN 2-METRIC SPACES AND THEIR APPLICATIONS
}

\author{
VINOD K. BHARDWAJ, VISHAL GUPTA, AND RAMAN DEEP
}

Received 10 October, 2014

\begin{abstract}
In this paper, we have given a criteria for the existence of common fixed point for pair of mappings in 2-metric spaces and establish common fixed point theorems for certain class of contractive type mappings. The results presented here, generalize some well known fixed point theorems given in the literature.
\end{abstract}

Keywords: 2-metric space, A-contractions, common fixed point, weakly compatible maps

\section{INTRODUCTION}

In the 1960's, Gahler introduced the notion of 2-metric space [9-11], whose abstract properties were suggested by the area of function in Euclidean space. Iseki [16] set out the tradition of proving fixed point theorems in 2-metric spaces employing various contractive conditions. Akram et al. [3,4] have introduced a large class of mappings called $A$-contractions, which is a proper superclass of Kannan's [18], Bianchini [6] and Reich [19] type contractions. Ahmad [1], Debashis Dey and Mantu Saha [7,20], Fathollahi [8] and Vishal Gupta [12] proved very useful common fixed point results in complete 2-metric spaces. Akram et al. [2,5] gave results for self maps satisfying $A$-contractions in the setting of $G$-metric spaces. Some other results [13-15] in different type of metric spaces are also proved as a generalization of Banach contraction principal.

\section{Preliminaries}

Definition 1 ([9]). Let $X$ be a non-empty set. A real valued function $d$ on $X \times$ $X \times X$ is said to be a 2-metric on $X$ if,

(i) for given distinct elements $x, y$ of $X$, there exist an element $z$ of $X$ such that $d(x, y, z) \neq 0$

(ii) $d(x, y, z)=0$, when at least two of $x, y, z$ are equal;

(iii) $d(x, y, z)=d(x, z, y)=d(y, z, x)$ for all $x, y, z$ in $X$;

(iv) $d(x, y, z) \leqslant d(x, y, w)+d(x, w, z)+d(w, y, z)$ for all $x, y, z, w$ in $X$.

This paper is in final form and no version of it will be submitted for publication elsewhere. 
When $d$ is a 2-metric on $X$, then the ordered pair $(X, d)$ is called a 2-metric space.

Definition 2 ([16]). A sequence $\left\{x_{n}\right\}$ in 2-metric space $X$ is said to be a Cauchy sequence, if for each $a \in X$,

$$
\lim _{n, m \rightarrow \infty} d\left(x_{n}, x_{m}, a\right)=0 .
$$

Definition 3 ([16]). A sequence $\left\{x_{n}\right\}$ in 2-metric space $X$ is said to be convergent to an element $x \in X$, if for each $a \in X$,

$$
\lim _{n \rightarrow \infty} d\left(x_{n}, x, a\right)=0 .
$$

Definition 4 ([17]). Two mappings $A, S$ are said to be weakly compatible in $X$ if they commute at their coincidence points i.e if for some $x \in X, A x=S x$, then $A S x=S A x$.

Akram et al. [3] defined $A$-contractions as follows:

Definition 5 ([3]). Let a non-empty set $A$ consisting of all functions $\alpha: \mathbb{R}_{+}^{3} \rightarrow \mathbb{R}_{+}$ satisfying:

(i) $\alpha$ is continuous on the set $\mathbb{R}_{+}^{3}$ of all triplets of non-negative reals (with respect to the Euclidean metric on $\mathbb{R}^{3}$ );

(ii) $a \leqslant k b$ for some $k \in[0,1)$, whenever $a \leqslant \alpha(a, b, b), a \leqslant \alpha(b, a, b)$ or $a \leqslant$ $\alpha(b, b, a)$ for all $a, b$.

Definition 6 ([20]). A self map $T$ on a 2-metric space $X$ is said to be $A$-contraction if for each $u \in X$,

$$
d(T x, T y, u) \leqslant \alpha[d(x, y, u), d(x, T x, u), d(y, T y, u)]
$$

holds for any $x, y \in X$ and some $\alpha \in A$.

In the present paper, we are proving some fixed point theorems for $A$-contraction mapping in a 2-metric spaces.

\section{MAIN RESULT}

Theorem 1. The self map $T$ on a 2-metric space $X$ satisfying

$$
\begin{gathered}
d(T x, T y, u) \leqslant \beta \max \{d(T x, x, u)+d(T y, y, u), d(T y, y, u)+ \\
d(x, y, u), d(T x, x, u)+d(x, y, u)\}
\end{gathered}
$$

for all $x, y, u$ in $X$ and some $\beta \in\left[0, \frac{1}{2}\right)$, is an A-contraction.

Proof. Define the map $\alpha: \mathbb{R}_{+}^{3} \rightarrow \mathbb{R}_{+}$as,

$$
\alpha(u, v, w) \leqslant \beta \max \{u+v, v+w, u+w\}
$$

for all $u, v, w$ in $\mathbb{R}_{+}$, where $\beta$ is any fixed number in $\left[0, \frac{1}{2}\right)$. Then $\alpha \in A$, because

(1) $\alpha$ is continuous; 
(2) for $u \leqslant \alpha(u, v, v)=\beta \max \{u+v, v+u, v+v\}$.

We consider the following cases:

Case I: Let $\max \{u+v, v+u, v+v\}=u+v$. In this case by the virtue of equation (3.1)

$$
u \leqslant \beta(u+v),
$$

that is,

$$
u \leqslant k v \text { with } k=\frac{\beta}{1-\beta} \in[0,1) \text {. }
$$

Case II: Let $\max \{u+v, v+u, v+v\}=v+v$. In this case $u \leqslant k v$, with $k=$ $2 \beta \in[0,1)$.

Similarly, when we have either $u \leqslant \alpha(v, u, v)$ or $u \leqslant(v, v, u)$, then $u \leqslant k v$ for some $k \in[0,1)$. Hence,

$$
\begin{aligned}
d(T x, T y, u) \leqslant & \beta \max \{d(T x, x, u)+d(T y, y, u), d(T y, y, u) \\
& +d(x, y, u), d(T x, x, u)+d(x, y, u)\} \\
= & \alpha\{d(x, y, u), d(T x, x, u), d(T y, y, u)\} .
\end{aligned}
$$

Thus $T$ is an $A$-contraction.

Theorem 2. Let $\alpha \in A$ and $\left\{T_{n}\right\}_{n=1}^{\infty}$ be a sequence of self maps on complete 2metric space $(X, d)$ such that

$$
d\left(T_{i} x, T_{j} y, u\right) \leqslant \alpha\left[d(x, y, u), d\left(T_{i} x, x, u\right), d\left(T_{j} y, y, u\right)\right]
$$

for all $x, y, u \in X$. Then $\left\{T_{n}\right\}_{n=1}^{\infty}$ has a unique common fixed point in $X$.

Proof. Consider $x_{0} \in X$ arbitrarily, then for each $n \in \mathbb{N}$, we define $x_{n}=T_{n} x_{n-1}$. Now,

$$
\begin{aligned}
d\left(x_{1}, x_{2}, u\right) & =d\left(T_{1} x_{0}, T_{2} x_{1}, u\right) \\
& \leqslant \alpha\left[d\left(x_{0}, x_{1}, u\right), d\left(T_{1} x_{0}, x_{0}, u\right), d\left(T_{2} x_{1}, x_{1}, u\right)\right] \\
& =\alpha\left[d\left(x_{0}, x_{1}, u\right), d\left(x_{1}, x_{0}, u\right), d\left(x_{2}, x_{1}, u\right)\right] \\
& \leqslant k d\left(x_{0}, x_{1}, u\right),
\end{aligned}
$$

for some $k \in[0,1)$ and $\alpha \in A$.

Again,

$$
\begin{aligned}
d\left(x_{2}, x_{3}, u\right) & =d\left(T_{2} x_{1}, T_{3} x_{2}, u\right) \\
& \leqslant \alpha\left[d\left(x_{1}, x_{2}, u\right), d\left(T_{2} x_{1}, x_{1}, u\right), d\left(T_{3} x_{2}, x_{2}, u\right)\right] \\
& =\alpha\left[d\left(x_{1}, x_{1}, u\right), d\left(x_{2}, x_{1}, u\right), d\left(x_{3}, x_{2}, u\right)\right] \\
& \leqslant k d\left(x_{1}, x_{2}, u\right) .
\end{aligned}
$$

We get from (3.3) and (3.4),

$$
d\left(x_{2}, x_{3}, u\right) \leqslant k^{2} d\left(x_{0}, x_{1}, u\right) .
$$


Proceeding in this way, we get

$$
d\left(x_{n}, x_{n+1}, u\right) \leqslant k^{n} d\left(x_{0}, x_{1}, u\right) .
$$

Next we prove that

$$
\begin{aligned}
d\left(x_{n}, x_{n+2}, u\right) & \leqslant d\left(x_{n}, x_{n+2}, x_{n+1}\right)+d\left(x_{n}, x_{n+1}, u\right)+d\left(x_{n+1}, x_{n+2}, u\right) \\
& \leqslant d\left(x_{n}, x_{n+2}, x_{n+1}\right)+\sum_{r=0}^{1} d\left(x_{n+r}, x_{n+r+1}, u\right) .
\end{aligned}
$$

Now,

$$
\begin{aligned}
d\left(x_{n}, x_{n+2}, x_{n+1}\right)= & d\left(x_{n+1}, x_{n+2}, x_{n}\right)=d\left(T_{n+1}\left(x_{n}\right), T_{n+2}\left(x_{n+1}\right), x_{n}\right) \\
\leqslant & \alpha\left[d\left(x_{n}, x_{n+1}, x_{n}\right), d\left(x_{n}, T_{n+1}\left(x_{n}\right), x_{n}\right),\right. \\
& \left.d\left(x_{n+1}, T_{n+2}\left(x_{n+1}\right), x_{n}\right)\right] \\
= & \alpha\left[d\left(x_{n}, x_{n+1}, x_{n}\right), d\left(x_{n}, x_{n+1}, x_{n}\right), d\left(x_{n+1}, x_{n+2}, x_{n}\right)\right] \\
\leqslant & k d\left(x_{n}, x_{n+1}, x_{n}\right) \text { for some } k \in[0,1) .
\end{aligned}
$$

Since $\alpha \in A$, so it follows that

$$
d\left(x_{n}, x_{n+2}, x_{n+1}\right)=0 .
$$

From (3.6) and (3.7), we get,

$$
d\left(x_{n}, x_{n+2}, u\right) \leqslant \sum_{r=0}^{1} d\left(x_{n+r}, x_{n+r+1}, u\right) .
$$

Again, by using the property (iv) of Definition 1,

$$
d\left(x_{n}, x_{n+3}, u\right) \leqslant \sum_{r=0}^{1} d\left(x_{n+3}, x_{n+r}, x_{n+r+1}\right)+\sum_{r=0}^{2} d\left(x_{n+r}, x_{n+r+1}, u\right) .
$$

Similarly, we can show that,

$$
d\left(x_{n+3}, x_{n}, x_{n+1}\right)=0 \quad \text { and } \quad d\left(x_{n+3}, x_{n+1}, x_{n+2}\right)=0 .
$$

Hence, $d\left(x_{n}, x_{n+3}, u\right) \leqslant \sum_{r=0}^{2} d\left(x_{n+2}, x_{n+r+1}, u\right)$. Continuing in the same sense, we get,

$$
d\left(x_{n}, x_{n+p}, u\right) \leqslant \sum_{r=0}^{p-1} d\left(x_{n+r}, x_{n+r+1}, u\right)
$$

for any integer $p$. So we have, for any integer $p>0$,

$$
d\left(x_{n}, x_{n+p}, u\right) \leqslant \frac{k^{n}}{1-k} d\left(x_{0}, x_{1}, u\right) .
$$


Hence $\left\{x_{n}\right\}$ is a Cauchy sequence in $X$ and so by completeness of $X,\left\{x_{n}\right\}$ converges to a point $z \in X$.

Now,

$$
\begin{aligned}
d\left(x_{n+1}, T_{n} z, u\right) & =d\left(T_{n+1}\left(x_{n}\right), T_{n}(z), u\right) \\
& \leqslant \alpha\left[d\left(x_{n}, z, u\right), d\left(x_{n}, T_{n+1}\left(x_{n}\right), u\right), d\left(z, T_{n} z, u\right)\right] \\
& =\alpha\left[d\left(x_{n}, z, u\right), d\left(x_{n}, x_{n+1}, u\right), d\left(z, T_{n} z, u\right)\right] .
\end{aligned}
$$

Taking limit as $n \rightarrow \infty$, we get

$$
\begin{aligned}
d\left(z, T_{n} z, u\right) & \leqslant \alpha\left[d(z, z, u), d(z, z, u), d\left(z, T_{n} z, u\right)\right] \\
& \leqslant k d(z, z, u) \\
& =0 .
\end{aligned}
$$

This gives $T_{n} z=z$.

To prove the uniqueness, let $w$ be another fixed point of $T_{n}$ for all $n$, then

$$
\begin{aligned}
d(z, w, u) & =d\left(T_{i} z, T_{j} w, u\right) \\
& \leqslant \alpha\left[d(z, w, u), d\left(z, T_{i} z, u\right), d\left(w, T_{j} w, u\right)\right] \\
& =\alpha[d(z, w, u), d(z, z, u), d(w, w, u)] \\
& =\alpha[d(z, w, u), 0,0] \\
& \leqslant k \cdot 0 \\
& =0 .
\end{aligned}
$$

This gives $z=w$.

Theorem 3. Let $X$ be a 2-metric space with 2-metrics $d$, and $\delta$ satisfying the following conditions,

(i) $d(x, y, z) \leqslant \delta(x, y, z)$;

(ii) $X$ is complete 2-metric space with respect to $d$;

(iii) $S, T$ are self maps on $X$ such that $T$ is continuous with respect to $d$ and

$$
\delta(T x, S y, u) \leqslant \alpha[\delta(x, y, u), \delta(x, T x, u), \delta(y, \delta y, u)]
$$

for all $x, y, u \in X$ and for some $\alpha \in A$.

Then $S$ and $T$ have a unique common fixed point.

Proof. For an arbitrary point $x_{0} \in X$, define a sequence $\left\{x_{n}\right\} \subset X$ as follows:

$$
T x_{2 n}=x_{2 n+1}, \quad S x_{2 n+1}=x_{2 n+2} \text {. }
$$

For every non-negative integer $n$, and for all $u \in X$,

$$
\delta\left(x_{2 n}, x_{2 n+1}, u\right)=\delta\left(S x_{2 n-1}, T x_{2 n}, u\right)=\delta\left(T x_{2 n}, S x_{2 n-1}, u\right),
$$

then by the above inequality (iii)

$$
\leqslant \alpha\left[\delta\left(x_{2 n}, x_{2 n-1}, u\right), \delta\left(x_{2 n}, T x_{2 n}, u\right), \delta\left(x_{2 n-1}, S x_{2 n-1}, u\right)\right]
$$




$$
\begin{aligned}
& =\alpha\left[\delta\left(x_{2 n}, x_{2 n-1}, u\right), \delta\left(x_{2 n}, x_{2 n+1}, u\right), \delta\left(x_{2 n-1}, x_{2 n}, u\right)\right] \\
& \leqslant k \delta\left(x_{2 n-1}, x_{2 n}, u\right) .
\end{aligned}
$$

Again consider,

$$
\begin{aligned}
\delta\left(x_{2 n-1}, x_{2 n}, u\right)= & \delta\left(T x_{2 n-2}, S x_{2 n-1}, u\right) \\
\leqslant & \alpha\left[\delta\left(x_{2 n-2}, x_{2 n-1}, u\right), \delta\left(x_{2 n-2}, T x_{2 n-2}, u\right),\right. \\
& \left.\delta\left(x_{2 n-1}, S x_{2 n-1}, u\right)\right] \\
= & \alpha\left[\delta\left(x_{2 n-2}, x_{2 n-1}, u\right), \delta\left(x_{2 n-2}, x_{2 n-1}, u\right),\right. \\
& \left.\delta\left(x_{2 n-1}, x_{2 n}, u\right)\right] . \\
\delta\left(x_{2 n-1}, x_{2 n}, u\right) \leqslant & k \delta\left(x_{2 n-2}, x_{2 n-1}, u\right) \quad \text { for some } k \in[0,1) .
\end{aligned}
$$

From (3.8) and (3.9), we conclude

$$
\delta\left(x_{2 n}, x_{2 n+1}, u\right) \leqslant k \delta\left(x_{2 n-1}, x_{2 n}, u\right) \leqslant k^{2} \delta\left(x_{2 n-2}, x_{2 n-1}, u\right) .
$$

Continuing in this way, we get

$$
\delta\left(x_{2 n}, x_{2 n+1}, u\right) \leqslant k^{2 n} \delta\left(x_{0}, x_{1}, u\right) .
$$

In general,

$$
\delta\left(x_{m}, x_{m+1}, u\right) \leqslant k^{m} \delta\left(x_{0}, x_{1}, u\right) \text { for all } u \in X .
$$

Now, we claim that $\delta\left(x_{0}, x_{1}, x_{n}\right)=0$ for all $n \in \mathbb{N}$. Clearly it is true for $n=0$ and $n=1$. Based on mathematical induction, let it be true for $1 \leqslant n \leqslant l-1$, then

$$
\begin{aligned}
\delta\left(x_{0}, x_{1}, x_{l}\right) & \leqslant \delta\left(x_{0}, x_{1}, x_{l-1}\right)+\delta\left(x_{0}, x_{l-1}, x_{l}\right)+\delta\left(x_{l-1}, x_{1}, x_{l}\right) \\
& =0+\delta\left(x_{l-1}, x_{l}, x_{0}\right)+\delta\left(x_{l-1}, x_{l}, x_{1}\right) \\
& \leqslant k^{l-1}\left[\delta\left(x_{0}, x_{1}, x_{0}\right)+\delta\left(x_{0}, x_{1}, x_{1}\right)\right] \\
& =0 .
\end{aligned}
$$

Thus we have

$$
\delta\left(x_{0}, x_{1}, x_{n}\right)=0 \text { for all } n \in \mathbb{N} .
$$

Hence (3.10) and (3.11) shows $\delta\left(x_{m}, x_{m+1}, x_{n}\right)=0$ for all $n, m \in \mathbb{N}$. Now, for $n>m$, we have

$$
\begin{aligned}
\delta\left(x_{m}, x_{n}, u\right) & \leqslant \delta\left(x_{m}, x_{m+1}, u\right)+\delta\left(x_{m}, x_{m+1}, x_{n}\right)+\delta\left(x_{m+1}, x_{n}, u\right) \\
& =\delta\left(x_{m}, x_{m+1}, u\right)+\delta\left(x_{m+1}, x_{n}, u\right) \\
& \leqslant \delta\left(x_{m}, x_{m+1}, u\right)+\delta\left(x_{m+1}, x_{m+2}, u\right)+\cdots+\delta\left(x_{n-1}, x_{n}, u\right) \\
& \leqslant\left(k^{m}+k^{m+1}+\cdots+k^{n-1}\right) \delta\left(x_{0}, x_{1}, u\right) \\
& \leqslant k^{m}\left(1+k+\cdots+k^{n-m-1}\right) \delta\left(x_{0}, x_{1}, u\right) \\
& \leqslant k^{m} \frac{\left(1-k^{n-m}\right)}{1-k} \delta\left(x_{0}, x_{1}, u\right) .
\end{aligned}
$$


Thus by condition (i),

$$
d\left(x_{n}, x_{m}, u\right) \leqslant \delta\left(x_{n}, x_{m}, u\right) \leqslant \frac{k^{m}\left(1-k^{n-m}\right)}{1-k} \delta\left(x_{0}, x_{1}, u\right),
$$

for all $n, m \in \mathbb{N}$ with $k \in[0,1)$.

So $\left\{x_{n}\right\}$ is a Cauchy sequence in $X$ with respect to $d$ and hence by condition (ii), $d\left(x_{n}, x^{\prime}, u\right) \rightarrow 0$ for some $x^{\prime} \in X$ and for each $u \in X$.

Since $T$ is given to be continuous with respect to $d$, we have,

$$
0=\lim _{n \rightarrow \infty} d\left(x_{2 n+1}, x^{\prime}, u\right)=\lim _{n \rightarrow \infty} d\left(T x_{2 n}, x^{\prime}, u\right)=d\left(T x^{\prime}, x^{\prime}, u\right)
$$

for all $u \in X$. So $T x^{\prime}=x^{\prime}$.

Now by condition (iii), for each $u \in X$

$$
\begin{aligned}
\delta\left(x^{\prime}, S x^{\prime}, u\right) & =\delta\left(T x^{\prime}, S x^{\prime}, u\right) \\
& \leqslant \alpha\left[\delta\left(x^{\prime}, x^{\prime}, u\right), \delta\left(x^{\prime}, T x^{\prime}, u\right), \delta\left(x^{\prime}, S x^{\prime}, u\right)\right] \\
& \leqslant \alpha\left[0,0, \delta\left(x^{\prime}, S x^{\prime}, u\right) .\right] \\
& <k \cdot 0 \\
& =0 .
\end{aligned}
$$

Hence, $x^{\prime}=S x^{\prime}$.

Thus $x^{\prime}$ is a common fixed point of $S$ and $T$. For the uniqueness, let $y$ be another common fixed point of $S$ and $T$ in $X$.

Then by condition (iii)

$$
\begin{aligned}
\delta\left(x^{\prime}, y, u\right) & =\delta\left(T x^{\prime}, S y, u\right) \\
& \leqslant \alpha\left[\delta\left(x^{\prime}, y, u\right), \delta\left(x^{\prime}, T x^{\prime}, u\right), \delta(y, S y, u)\right] \\
& \leqslant 0 .
\end{aligned}
$$

Thus, $x^{\prime}=y$.

\section{Applications}

Here, we give some applications related to our results. For this we use a Lebesgue integrable function as a summable for each compact $R^{+}$.

Let us define $\psi:[0, \infty) \rightarrow[0, \infty)$ as $\psi(t)=\int_{0}^{t} \varphi(t), \forall t>0$ be a non-decreasing and continuous function. Moreover, for each $\epsilon>0, \varphi(\epsilon)>0$ and $\varphi(t)=0$ iff $t=0$. 
Theorem 4. Let $\left\{T_{n}\right\}_{n=1}^{\infty}$ be a sequence of self maps on the complete 2-metric space $(X, d)$ satisfying the following condition:

$$
\begin{aligned}
& \int_{0}^{d\left(T_{i} x, T_{j} y, u\right)} \varphi(t) d t \\
& \leq \alpha\left(\int_{0}^{d(x, y, u)} \varphi(t) d t, \int_{0}^{d\left(T_{i} x, x, u\right)} \varphi(t) d t, \int_{0}^{d\left(T_{j} y, y, u\right)} \varphi(t) d t\right)
\end{aligned}
$$

for some $x, y \in X$ with some $\alpha \in A$, where $\varphi:[0, \infty) \rightarrow[0, \infty)$ is a Lebesgueintegrable mapping which is summable(i.e with finite integral) on each compact subset of $[0, \infty)$, non-negative and such that for each $\epsilon>0$,

$$
\int_{0}^{\epsilon} \varphi(t) d t>0
$$

Then $\left\{T_{n}\right\}_{n=1}^{\infty}$ has a unique fixed point in $X$.

Proof. Let $x_{0} \in X$ be arbitrary and for each $n \in N$ define $x_{n}=T_{n} x_{n-1}$. Now

$$
\begin{aligned}
& \int_{0}^{d\left(x_{1}, x_{2}, u\right)} \varphi(t) d t=\int_{0}^{d\left(T_{1} x_{0}, T_{2} x_{1}, u\right)} \varphi(t) d t \\
& \leq \alpha\left(\int_{0}^{d\left(x_{0}, x_{1}, u\right)} \varphi(t) d t, \int_{0}^{d\left(T_{1} x_{0}, x_{0}, u\right)} \varphi(t) d t, \int_{0}^{d\left(T_{2} x_{1}, x_{1}, u\right)} \varphi(t) d t\right) \\
& =\alpha\left(\int_{0}^{d\left(x_{0}, x_{1}, u\right)} \varphi(t) d t, \int_{0}^{d\left(x_{1}, x_{0}, u\right)} \varphi(t) d t, \int_{0}^{d\left(x_{2}, x_{1}, u\right)} \varphi(t) d t\right) .
\end{aligned}
$$

By definition of $\alpha$, we get

$$
\int_{0}^{d\left(x_{1}, x_{2}, u\right)} \varphi(t) \leq k \int_{0}^{d\left(x_{0}, x_{1}, u\right)} \varphi(t)
$$

for some $k \in[0,1)$ as $\alpha \in A$.

Again,

$$
\begin{aligned}
& \int_{0}^{d\left(x_{2}, x_{3}, u\right)} \varphi(t)=\int_{0}^{d\left(T_{2} x_{1}, T_{3} x_{2}, u\right)} \varphi(t) \\
& \leq \alpha\left(\int_{0}^{d\left(x_{1}, x_{2}, u\right)} \varphi(t) d t, \int_{0}^{d\left(T_{2} x_{1}, x_{1}, u\right)} \varphi(t) d t, \int_{0}^{d\left(T_{3} x_{2}, x_{2}, u\right)} \varphi(t) d t\right) \\
& =\alpha\left(\int_{0}^{d\left(x_{1}, x_{2}, u\right)} \varphi(t) d t, \int_{0}^{d\left(x_{2}, x_{1}, u\right)} \varphi(t) d t, \int_{0}^{d\left(x_{3}, x_{2}, u\right)} \varphi(t) d t\right) .
\end{aligned}
$$


Therefore,

$$
\int_{0}^{d\left(x_{2}, x_{3}, u\right)} \varphi(t) \leq k \int_{0}^{d\left(x_{1}, x_{2}, u\right)} \varphi(t)
$$

from equation (4.3) and (4.4), we get

$$
\int_{0}^{d\left(x_{2}, x_{3}, u\right)} \varphi(t) \leq k^{2} \int_{0}^{d\left(x_{0}, x_{1}, u\right)} \varphi(t)
$$

Proceeding in the same way, we have

$$
\int_{0}^{d\left(x_{n}, x_{n+1}, u\right)} \varphi(t) \leq k^{n} \int_{0}^{d\left(x_{0}, x_{1}, u\right)} \varphi(t) .
$$

Taking limit as $n \rightarrow \infty$, we get $\lim _{n} \int_{0}^{d\left(x_{n}, x_{n+1}, u\right)} \varphi(t) d t=0, \quad$ as $k \in[0,1)$, which from (4.2) implies that $\lim _{n} d\left(x_{n}, x_{n+1}, u\right)=0$.

We now show that $\left\{x_{n}\right\}$ is a Cauchy sequence.

\section{Consider,}

$d\left(x_{n}, x_{n+2}, u\right) \leq d\left(x_{n}, x_{n+2}, x_{n+1}\right)+d\left(x_{n}, x_{n+1}, u\right)+d\left(x_{n+1}, x_{n+2}, u\right)$.

Therefore,

$$
d\left(x_{n}, x_{n+2}, u\right) \leq d\left(x_{n}, x_{n+2}, x_{n+1}\right)+\sum_{r=0}^{1} d\left(x_{n+r}, x_{n+r+1}, u\right) .
$$

Now

$$
\begin{gathered}
\int_{0}^{d\left(x_{n}, x_{n+2}, x_{n+1}\right)} \varphi(t) d t=\int_{0}^{d\left(x_{n+1}, x_{n+2}, x_{n}\right)} \varphi(t) d t \\
=\int_{0}^{d\left(T_{n+1}\left(x_{n}\right), T_{n+2}\left(x_{n+1}\right), x_{n}\right)} \varphi(t) d t \\
\leq \alpha\left(\int_{0}^{d\left(x_{n}, x_{n+1}, x_{n}\right)} \varphi(t) d t\right.
\end{gathered}
$$




$$
\begin{gathered}
\left.\int_{0}^{d\left(x_{n}, T_{n+1}\left(x_{n}\right), x_{n}\right)} \varphi(t) d t, \int_{0}^{d\left(x_{n+1}, T_{n+2}\left(x_{n+1}\right), x_{n}\right)} \varphi(t) d t\right) \\
=\alpha\left(\int_{0}^{d\left(x_{n}, x_{n+1}, x_{n}\right)} \varphi(t) d t, \int_{0}^{d\left(x_{n}, x_{n+1}, x_{n}\right)} \varphi(t) d t, \int_{0}^{d\left(x_{n+1}, x_{n+2}, x_{n}\right)} \varphi(t) d t\right) \\
\leq k \int_{0}^{d\left(x_{n}, x_{n+1}, x_{n}\right)} \varphi(t) d t \text { for } k \in[0,1) \text { and } \alpha \in A .
\end{gathered}
$$

It follows that $\int_{0}^{d\left(x_{n}, x_{n+2}, x_{n+1}\right)} \varphi(t) d t=0$,

and by $(4.2), d\left(x_{n}, x_{n+2}, x_{n+1}\right)=0$.

Again, by using the property (iv) of Definition 1,

$d\left(x_{n}, x_{n+3}, u\right)=\sum_{r=0}^{1} d\left(x_{n+3}, x_{n+r}, x_{n+r+1}\right)+\sum_{r=0}^{2} d\left(x_{n+r}, x_{n+r+1}, u\right)$.

As above, we can show that $d\left(x_{n+3}, x_{n}, x_{n+1}\right)=0$ and $d\left(x_{n+3}, x_{n+1}, x_{n+2}\right)=0$, hence

$d\left(x_{n}, x_{n+3}, u\right)=\sum_{n=0}^{2} d\left(x_{n+r}, x_{n+r+1}, u\right)$.

Continuing in the same manner, we get for any integer $p$,

$d\left(x_{n}, x_{n+p}, u\right) \leq \sum_{r=0}^{p-1} d\left(x_{n+r}, x_{n+r+1}, u\right) \leq \frac{k^{n}}{1-k} d\left(x_{0}, x_{1}, u\right) \rightarrow 0$

as $n \rightarrow \infty$, since $k \in[0,1)$.

Hence $\left\{x_{n}\right\}$ is a Cauchy sequence in $X$ and so by completeness of $X,\left\{x_{n}\right\}$ converges to a point $z \in X$.

Now, $\int_{0}^{d\left(x_{n+1}, T_{n} z, u\right)} \varphi(t) d t=\int_{0}^{d\left(T_{n+1}\left(x_{n}\right), T_{n} z, u\right)} \varphi(t) d t$

$$
\begin{aligned}
& \leq \alpha\left(\int_{0}^{d\left(x_{n}, z, u\right)} \varphi(t) d t, \int_{0}^{d\left(x_{n}, T_{n+1}\left(x_{n}\right), u\right)} \varphi(t) d t, \int_{0}^{d\left(z, T_{n} z, u\right)} \varphi(t) d t\right) \\
& =\alpha\left(\int_{0}^{d\left(x_{n}, z, u\right)} \varphi(t) d t, \int_{0}^{d\left(x_{n}, x_{n+1}, u\right)} \varphi(t) d t, \int_{0}^{d\left(z, T_{n} z, u\right)} \varphi(t) d t\right) .
\end{aligned}
$$

Taking limit as $n \rightarrow \infty$, we have

$\int_{0}^{d\left(z, T_{n} z, u\right)} \varphi(t) d t$ 
$\leq \alpha\left(\int_{0}^{d(z, z, u)} \varphi(t) d t, \int_{0}^{d(z, z, u)} \varphi(t) d t, \int_{0}^{d\left(z, T_{n} z, u\right)} \varphi(t) d t\right)$

$\leq k \int_{0}^{d(z, z, u)} \varphi(t)$

for some $k \in[0,1)$ as $\alpha \in A \Rightarrow \int_{0}^{d\left(z, T_{n} z, u\right)} \varphi(t) d t=0$,

and by (4.2), $d\left(z, T_{n} z, u\right)=0$ and thus $T_{n} z=z \forall n$.

To prove uniqueness of $z$, let $w$ be another fixed point of $T_{n}$, Then

$$
\begin{aligned}
& \int_{0}^{d(z, w, u)} \varphi(t) d t=\int_{0}^{d\left(T_{i} z, T_{j} w, u\right)} \varphi(t) d t \\
& \leq \alpha\left(\int_{0}^{d(z, w, u)} \varphi(t) d t, \int_{0}^{d\left(z, T_{i} z, u\right)} \varphi(t) d t, \int_{0}^{d\left(w, T_{j} w, u\right)} \varphi(t) d t\right) \\
& =\alpha\left(\int_{0}^{d(z, w, u)} \varphi(t) d t, \int_{0}^{d(z, z, u)} \varphi(t) d t, \int_{0}^{d(w, w, u)} \varphi(t) d t\right) \\
& =\alpha\left(\int_{0}^{d(z, w, u)} \varphi(t) d t, 0,0\right) \leq k .0=0
\end{aligned}
$$

for some $k \in[0,1)$ as $\alpha \in A$. This gives $z=w$.

Theorem 5. Let $X$ be a set with 2-metrics $d$ and $\delta$ satisfying the following conditions:

(i) $d(x, y, z) \leq \delta(x, y, z)$;

(ii) $X$ is complete 2-metric space with respect to $d$;

(iii) $S$ and $T$ are self maps on $X$ such that $T$ is continuous with respect to $d$ and satisfying

$$
\begin{aligned}
& \int_{0}^{\delta(T x, S y, u)} \varphi(t) d t \leq \\
& \alpha\left[\int_{0}^{\delta(x, y, u)} \varphi(t) d t, \int_{0}^{\delta(x, T x, u)} \varphi(t) d t, \int_{0}^{\delta(y, S y, u)} \varphi(t) d t\right]
\end{aligned}
$$

for all $x, y, u \in X$ and for some $\alpha \in A$, where $\varphi:[0, \infty) \rightarrow[0, \infty)$ is a Lebesgue integrable mapping which is summable (i.e finite integral) on each compact subset of $[0, \infty)$, non-negative and such that for each $\epsilon>0, \int_{0}^{\epsilon} \varphi(t) d t>0$.

Then $S$ and $T$ have a unique common fixed point.

Proof. For an arbitrary point $x_{0} \in X$, define a sequence $\left\{x_{n}\right\} \subset X$ as follows:-

$T x_{2 n}=x_{2 n+1}, S x_{2 n+1}=x_{2 n+2}$ for every non-negative integer $n$ and for all $u \in X$.

Now $\int_{0}^{\delta\left(x_{2 n}, x_{2 n+1}, u\right)} \varphi(t) d t=\int_{0}^{\delta\left(S x_{2 n-1}, T x_{2 n}, u\right)} \varphi(t) d t$ 


$$
\begin{aligned}
& =\int_{0}^{\delta\left(T x_{2 n}, S x_{2 n-1}, u\right)} \varphi(t) d t \\
& \leq \alpha\left[\int_{0}^{\delta\left(x_{2 n}, x_{2 n-1}, u\right)} \varphi(t) d t, \int_{0}^{\delta\left(x_{2 n}, T x_{2 n}, u\right)} \varphi(t) d t, \int_{0}^{\delta\left(x_{2 n-1}, S x_{2 n-1}, u\right)} \varphi(t) d t\right] \\
& \leq \alpha\left[\int_{0}^{\delta\left(x_{2 n}, x_{2 n-1}, u\right)} \varphi(t) d t, \int_{0}^{\delta\left(x_{2 n}, x_{2 n-1}, u\right)} \varphi(t) d t, \int_{0}^{\delta\left(x_{2 n-1}, x_{2 n}, u\right)} \varphi(t) d t\right] .
\end{aligned}
$$

Thus,

$$
\int_{0}^{\delta\left(x_{2 n}, x_{2 n+1}, u\right)} \varphi(t) d t \leq k \int_{0}^{\delta\left(x_{2 n-1}, x_{2 n}, u\right)} \varphi(t) d t
$$

for some $k \in[0,1)$ as $\alpha \in A$.

Consider, $\int_{0}^{\delta\left(x_{2 n-1}, x_{2 n}, u\right)} \varphi(t) d t=\int_{0}^{\delta\left(T x_{2 n-2}, S x_{2 n-1}, u\right)} \varphi(t) d t$

$\leq \alpha\left[\int_{0}^{\delta\left(x_{2 n-2}, x_{2 n-1}, u\right)} \varphi(t) d t, \int_{0}^{\delta\left(x_{2 n-2}, T x_{2 n-2}, u\right)} \varphi(t) d t\right.$,

$\left.\int_{0}^{\delta\left(x_{2 n-1}, S x_{2 n-1}, u\right)} \varphi(t) d t\right]$

$\leq \alpha\left[\int_{0}^{\delta\left(x_{2 n-2}, x_{2 n-1}, u\right)} \varphi(t) d t, \int_{0}^{\delta\left(x_{2 n-2}, x_{2 n-1}, u\right)} \varphi(t) d t, \int_{0}^{\delta\left(x_{2 n-1}, x_{2 n}, u\right)} \varphi(t) d t\right]$.

By definition of function $\alpha$,

$$
\int_{0}^{\delta\left(x_{2 n-1}, x_{2 n}, u\right)} \varphi(t) d t \leq k \int_{0}^{\delta\left(x_{2 n-2}, x_{2 n-1}, u\right)} \varphi(t) d t
$$

for some $k \in[0,1)$.

From (4.8) and (4.9), we conclude that

$\int_{0}^{\delta\left(x_{2 n}, x_{2 n+1}, u\right)} \varphi(t) d t \leq k \int_{0}^{\delta\left(x_{2 n-1}, x_{2 n}, u\right)} \varphi(t) d t \leq k^{2} \int_{0}^{\delta\left(x_{2 n-2}, x_{2 n-1}, u\right)} \varphi(t) d t$.

Proceeding in the same way, we get

$\int_{0}^{\delta\left(x_{2 n}, x_{2 n+1}, u\right)} \varphi(t) d t \leq k^{2 n} \int_{0}^{\delta\left(x_{0}, x_{1}, u\right)} \varphi(t) d t$.

In general,

$$
\int_{0}^{\delta\left(x_{m}, x_{m+1}, u\right)} \varphi(t) d t \leq k^{m} \int_{0}^{\delta\left(x_{0}, x_{1}, u\right)} \varphi(t) d t, \quad \forall u \in X .
$$

Now we claim that $\delta\left(x_{0}, x_{1}, x_{n}\right)=0$ for all $n \in N$. Clearly it is true for $n=0$ and $n=1$, let it be true for $1 \leq n \leq l-1$, then

$\delta\left(x_{0}, x_{1}, x_{l}\right) \leq \delta\left(x_{0}, x_{1}, x_{l-1}\right)+\delta\left(x_{0}, x_{l-1}, x_{l}\right)+\delta\left(x_{l-1}, x_{1}, x_{l}\right)$ 


$$
\begin{aligned}
& =0+\delta\left(x_{l-1}, x_{l}, x_{0}\right)+\delta\left(x_{l-1}, x_{l}, x_{1}\right) \\
& \leq k^{l-1}\left[\delta\left(x_{0}, x_{1}, x_{0}\right)+\delta\left(x_{0}, x_{1}, x_{1}\right)\right] .
\end{aligned}
$$

Thus we have,

$$
\delta\left(x_{0}, x_{1}, x_{n}\right)=0 \text { for all } n \in N .
$$

Hence (4.10) and (4.11) implies, $\delta\left(x_{m}, x_{m+1}, x_{n}\right)=0$.

$$
\begin{aligned}
& \text { Consider, } \int_{0}^{\delta\left(x_{m}, x_{n}, u\right)} \varphi(t) d t \\
\leq & \int_{0}^{\delta\left(x_{m}, x_{m+1}, u\right)} \varphi(t) d t+\int_{0}^{\delta\left(x_{m}, x_{m+1}, x_{n}\right)} \varphi(t) d t+\int_{0}^{\delta\left(x_{m+1}, x_{n}, u\right)} \varphi(t) d t \\
= & \int_{0}^{\delta\left(x_{m}, x_{m+1}, u\right)} \varphi(t) d t+\int_{0}^{\delta\left(x_{m+1}, x_{n}, u\right)} \varphi(t) d t \\
\leq & \int_{0}^{\delta\left(x_{m}, x_{m+1}, u\right)} \varphi(t) d t+\int_{0}^{\delta\left(x_{m+1}, x_{m+2}, u\right)} \varphi(t) d t+\ldots+\int_{0}^{\delta\left(x_{n-1}, x_{n}, u\right)} \varphi(t) d t \\
\leq & \left(k^{m}+k^{m-1}+\ldots+k^{n-1}\right) \int_{0}^{\delta\left(x_{0}, x_{1}, u\right)} \varphi(t) d t \\
= & \frac{k^{m}\left(1-k^{n-m}\right)}{1-k} \int_{0}^{\delta\left(x_{0}, x_{1}, u\right)} \varphi(t) d t \text { for some } k \in[0,1) .
\end{aligned}
$$

Taking $n, m \rightarrow \infty$, we have $\int_{0}^{\delta\left(x_{n}, x_{m}, u\right)} \varphi(t) d t=0$, and then $\delta\left(x_{n}, x_{m}, u\right)=0$ as $n, m \rightarrow \infty$.

Thus by condition (i), $d\left(x_{n}, x_{m}, u\right) \leq \delta\left(x_{n}, x_{m}, u\right) \rightarrow 0$ as $n, m \rightarrow \infty$.

So $\left\{x_{n}\right\}$ is a Cauchy sequence in $X$ with respect to $d$ and hence $d\left(x_{n}, x^{\prime}, u\right) \rightarrow 0$ for some $x^{\prime} \in X$ as $n \rightarrow \infty$ and for each $u \in X$. Since $T$ is given to be continuous with respect to $d$, we have

$$
0=\lim _{n \rightarrow \infty} d\left(x_{2 n+1}, x^{\prime}, u\right)=\lim _{n \rightarrow \infty} d\left(T x_{2 n}, x^{\prime}, u\right)=d\left(T x^{\prime}, x^{\prime}, u\right) \text { for all }
$$
$u \in X$, so that $T x^{\prime}=x^{\prime}$.

From condition (iii), for each $u \in X$ we have,

$$
\begin{aligned}
& \int_{0}^{\delta\left(x^{\prime}, S x^{\prime}, u\right)} \varphi(t) d t=\int_{0}^{\delta\left(T x^{\prime}, S x^{\prime}, u\right)} \varphi(t) d t \\
\leq & \alpha\left[\int_{0}^{\delta\left(x^{\prime}, x^{\prime}, u\right)} \varphi(t) d t, \int_{0}^{\delta\left(x^{\prime}, T x^{\prime}, u\right)} \varphi(t) d t, \int_{0}^{\delta\left(x^{\prime}, S x^{\prime}, u\right)} \varphi(t) d t\right]
\end{aligned}
$$


$\leq \alpha\left[0,0, \int_{0}^{\delta\left(x^{\prime}, S x^{\prime}, u\right)} \varphi(t)\right] \leq k .0=0 \quad$ for some $k \in[0,1)$

and hence $\delta\left(x^{\prime}, S x^{\prime}, u\right)=0$ for all $u \in X$.

Therefore $x^{\prime}=S x^{\prime}$. Thus $x^{\prime}$ is the common fixed point of $S$ and $T$.

For uniqueness, let $y$ be any other common fixed point of $S$ and $T$ in $X$, then by condition (iii),

$$
\begin{gathered}
\int_{0}^{\delta\left(x^{\prime}, y, u\right)} \varphi(t) d t=\int_{0}^{\delta\left(T x^{\prime}, S y, u\right)} \varphi(t) d t \\
\leq \alpha\left[\int_{0}^{\delta\left(x^{\prime}, y, u\right)} \varphi(t) d t, \int_{0}^{\delta\left(x^{\prime}, T x^{\prime}, u\right)} \varphi(t) d t, \int_{0}^{\delta(y, S y, u)} \varphi(t) d t\right] .
\end{gathered}
$$

Hence $x^{\prime}=y$.

Example 1. Consider $X=\{1,2,3,4\}$ and define $d: X \times X \times X \rightarrow R$ by $d(x, y, z)=$ 0 for at least two of any $x, y, z$ are zero and $d(x, y, z)=d(y, x, z)=d(z, y, x)$ for $x \neq y \neq z$ be such that $d(1,2,3)=6, d(1,2,4)=7, d(1,3,4)=8, d(2,3,4)=9$. Then $(X, d)$ is 2 -metric space.

Let $T: X \rightarrow X$ be defined by $T(1)=2, T(2)=3, T(3)=4, T(4)=1$.

Then, clearly the condition of $A$-contraction

$d(T x, T y, u) \leq \alpha[d(x, y, u), d(x, T x, u), d(y, T y, u)]$ is not satisfied.

Take $x=1, y=2$ and $u=4$ L.H.S of condition becomes $d(T(1), T(2), 4)=$ $d(2,3,4)=9$ and R.H.S is

$$
\alpha[d(1,2,4), d(1, T(1), 4), d(2, T(2), 4)]=\alpha[7,7,9] .
$$

Then $9 \leq k .7$, this is a contradiction, as $k \in[0,1]$. Hence $T$ is not an $A$-contraction and $T$ has no fixed point.

Example 2. Consider 2-metric space as in Example 1, and define $T(1)=1, T(2)=1, T(3)=1, T(4)=1$. Clearly, the map $T$ satisfies all the conditions of Theorem 1, in all cases. Therefore $T$ is an $A$-contraction.

\section{REFERENCES}

[1] A. Ahmad, "The convergence of sequence of fixed points in 2-metric spaces," International Research Journal of Computer Science, vol. 3, no. 2, pp. 1-3, 2015.

[2] M. Akram and Nosheen, "Some fixed point theorems of A-type contrations in G-metric spaces," International Journal of Pure and Applied Mathematics, vol. 79, no. 2, pp. 219-233, 2012. 
[3] M. Akram and A. A. Siddique, "A fixed point theorem for A-contractions on a class of generalized metric spaces," Korean J. Math. Sciences, vol. 10, no. 2, pp. 1-5, 2003.

[4] M. Akram, A. A. Zafar, and A. A. Siddique, "A general class of contractions: A-contractions," Novi Sad J. Math., vol. 38, no. 1, pp. 25-33, 2008.

[5] M. Akram, A. A. Zafar, and A. A. Siddique, "Common fixed point theorems for self maps of a generalized metric space satisfying A-contration type condition," Int. Journal of Math. Analysis, vol. 5, no. 16, pp. 757-763, 2011.

[6] R. Bianchini, "Su un problema di s. reich riguar dante la teori dei punt i fissi," Bell Un. Math. Ital., vol. 5, pp. 103-108, 1972.

[7] D. Dey and M. Saha, "Common fixed point theorems in a complete 2-metric space," Acta Univ. Palacki. Olomuc., Fac. rer. nat., Mathematica, vol. 52, no. 1, pp. 79-87, 2013.

[8] S. Fathollahii, N. Hussain, and L. A. Khan, "Fixed point results for modified weak and rational $\alpha-\psi$-contractions in ordered 2-metric spaces," Fixed Point Theory and Applications, vol. 6, 2014.

[9] S. Gähler, "2-metrische raume and ihre topologische strucktur," Math. Nachr., vol. 26, pp. 115148, 1963, doi: 10.1002/mana.19630260109.

[10] S. Gähler, "Uber die uniformisierbakait 2-metrische raume," Math. Nachr., vol. 28, pp. 235-244, 1965, doi: 10.1002/mana.19640280309.

[11] S. Gähler, "Zur geometric 2-metrische raume," Rev. Raum. Math. Pures at Appl., vol. 11, pp. 655-664, 1966.

[12] V. Gupta and R. Kaur, "Some common fixed point theorems for a class of A-contractions on 2metric spaces," International Journal of Pure and Applied Mathematics, vol. 78, no. 6, pp. 909916, 2012.

[13] V. Gupta and R. Kaur, "Some coupled fixed point theorems in partially ordered s-metric spaces," Miskolc Mathematical Notes, vol. 16, no. 1, pp. 181-194, 2015.

[14] V. Gupta and N. Mani, "Existence and uniqueness of fixed point for contractive mapping of integral type," International Journal of Computing Science and Mathematics, vol. 4, no. 1, pp. 72 - 83, 2013, doi: 10.1504/IJCSM.2013.054685.

[15] V. Gupta, N. Mani, and A. K. Tripathi, "A fixed point theorem satisfying a generalized weak contractive condition of integral type," International Journal of Mathematical Analysis, vol. 6, pp. $1883-1889,2012$.

[16] K. Iseki, "Fixed point theorems in 2-metric spaces," Math. Sem. Notes., Kobe. Univ., vol. 3, pp. 133-136, 1975.

[17] G. Jungck, "Common fixed points for non-continuous non-self maps on non-metric spaces," Far East J. Math. Sci., vol. 4, no. 2, pp. 199-215, 1996.

[18] R. Kannan, “Some results on fixed points-II," Amer. Math. Monthly, vol. 76, no. 4, pp. 405-408, 1969, doi: $10.2307 / 2316437$.

[19] S. Reich, "Kannan's fixed point theorems," Ball. Un. Math. Ital., vol. 4, pp. 1-11, 1971.

[20] M. Saha and D. Dey, "Fixed point theorems for a class of A-contractions on a 2-metric space," Novi Sad J. Math., vol. 40, no. 1, pp. 3-8, 2010.

Authors' addresses

\section{Vinod K. Bhardwaj}

Department of Mathematics, Kurukshetra University, Kurukshetra, Haryana (India)

E-mail address: vinodk_bhj@erediffmail.com

\section{Vishal Gupta}

Department of Mathematics, Maharishi Markandeshwar University, Ambala, Haryana, (India)

E-mail address: vishal.gmnegmail.com 


\section{Raman Deep}

Department of Mathematics, Maharishi Markandeshwar University, Mullana, Ambala, Haryana, (India)

E-mail address: ramandeepvirk02@gmail.com 